\title{
METABOLISMO ENERGÉTICO DE Cucurbita pepo MICORRIZADA CON HONGOS DEL DESIERTO SONORENSE Y CRECIDA CON SALINIDAD O DÉFICIT DE HUMEDAD
}

\author{
ENERGY METABOLISM OF Cucurbita pepo MYCORRHIZED WITH FUNGI OF THE SONORAN DESERT \\ AND GROWN WITH SALINITY OR MOISTURE DEFICIT
}

\author{
Citlalli Harris-Valle ${ }^{1}$, Martín Esqueda $^{1 \star}$, Antonio Orozco Avitia ${ }^{1}$, Alejandro E. Castellanos ${ }^{2}$, Alfonso Gardea ${ }^{1}$ y \\ Elisa M. Valenzuela-Soto ${ }^{1}$
}

${ }^{1}$ Centro de Investigación en Alimentación y Desarrollo, A.C. Apdo. Postal 1735. 83000, Hermosillo, Sonora, México. Tel y Fax: 01 (662) 280-04-22. ${ }^{2}$ Universidad de Sonora. Apdo. Postal 54. 83000, Hermosillo, Sonora, México.

*Autor para correspondencia (esqueda@ciad.mx)

\section{RESUMEN}

Los hongos micorrízicos arbusculares (HMA) son útiles en la agricultura porque mejoran el crecimiento de las plantas, sobre todo en ambientes subóptimos. Debido a que la micorrización es una simbiosis obligada, se considera que la asociación es exitosa cuando el costo de mantener al hongo por la transferencia de fotoasimilados es compensado por los beneficios nutricionales que obtiene la planta. La eficiencia en la interacción cambia en función de la región de origen de los organismos asociados y de las condiciones de cultivo. En este trabajo se evaluó el efecto de HMA, procedentes de suelo salino en una región semiárida, en el crecimiento, costos de construcción y fotoquímica de plantas de Cucurbita pepo var. pepo crecidas con estrés salino y déficit de humedad. Los HMA originarios del desierto Sonorense incrementaron la biomasa de las plantas con menor costo de construcción del tejido vegetal, en comparación con una especie exótica (Glomus claroideum), con una fertilización química recomendada y con un testigo no inoculado ni fertilizado. Los beneficios observados están relacionados con el desarrollo del micelio extracelular y el porcentaje de colonización micorrízica. La micorrización de especies nativas incrementa los índices de valoración fotoquímica (flujo de fotones absorbidos por unidad de área y eficiencia con que un electrón es transferido a la cadena de transporte de electrones), respecto a la inoculación con una especie exótica o fertilización química.

Palabras clave: Cucurbita pepo, costos de construcción, déficit de humedad, fluorescencia, micorrización, salinidad.

\section{SUMMARY}

Arbuscular mycorrhizal fungi (AMF) are useful in agriculture for enhancing plant growth mainly in suboptimal environments. Because mycorrhizal symbiosis is required, a successful association occurs when the cost of maintaining the fungus by the transfer of photoassimilates is compensated by the nutritional benefits obtained by plants. The efficiency in this interaction changes depending on the origin of associated organisms and growing conditions. This study evaluated AMF effects collected from a saline soil of a semiarid region, on plant growth, construction costs and photochemistry of Cucurbita pepo var. pepo under saline stress and water deficit. AMF native of the Sonoran desert increased plant biomass with lower construction cost the plant tissue, compared to an exotic species (Glomus claroideum), to a recommended chemical fertilization and to a control. The observed benefits are related to development of extracellular mycelium and percentage of mycorrhizal colonization. Also, under moisture deficit and salinity, mycorrhization using native species increases the photochemistry valuation indexes (absorbed photon flux per unit area and efficiency by which an electron is transferred through the transport chain), as compared to plants inoculated with exotic species or fertilized chemically.

Index words: Cucurbita pepo, construction cost, water deficit, fluorescence, mycorrhization, salinity.

\section{INTRODUCCIÓN}

La simbiosis micorrízica es una asociación planta-hongo importante para la agricultura sostenible, ya que modifica el estado nutricional de las plantas. Por ello, en los últimos años se ha evaluado el potencial de los hongos micorrízicos arbusculares (HMA) para la producción de biofertilizantes (Lekberg y Koide, 2005; Smith y Read, 2008). Los HMA son simbiontes obligados y se calcula que de 3 a $4 \%$ de los fotoasimilados de la planta son transferidos al hongo (Smith y Read, 2008); pero existen estudios que demuestran que la demanda energética por efecto de la micorrización es compensada con la reducción de otros costos metabólicos, como la asimilación y utilización de fósforo (Black et al., 2000).

La eficiencia en términos de carbono (C) ganado como respuesta a la simbiosis y el utilizado en el mantenimiento del huésped es un modelo desarrollado con base en los conceptos de costo y beneficio. Si la planta transfiere C al hongo, pero aumenta su tasa de fijación por unidad de área y disminuye los requerimientos para producir o mantener una unidad de área fotosintética, entonces podrá tener mayor tasa de producción de fotoasimilados y posiblemente de crecimiento, en comparación con una planta no micorrizada (Tinker et al., 1994; Lapointe y Molard, 1997; Wright et al., 1998). 
La habilidad de un determinado HMA para inducir mayor resistencia al estrés por déficit de humedad o salinidad está directamente relacionada con un menor efecto en el crecimiento del hospedero bajo las condiciones estresantes (Tinker et al., 1994; Bhoopander y Mukerji, 2004). De modo que la modificación de parámetros fisiológicos en la planta por una especie de HMA, debe tomarse en cuenta para seleccionarla (Ruiz-Lozano, 2003). La fluorescencia de la clorofila es una evaluación adecuada de la fotosíntesis y es usada como respuesta fisiológica de las plantas micorrizadas sometidas a estrés (Zubek et al., 2009).

En los últimos años se ha visto que las especies de HMA nativas de regiones semiáridas son más eficientes para mejorar el metabolismo y crecimiento de las plantas con estrés hídrico (Cho et al., 2005; García et al., 2008). En el presente estudio se evaluaron los efectos de HMA nativos del desierto Sonorense en el crecimiento, micorrización, fotoquímica de las plantas y costo de construcción de Cucurbita pepo L. var. pepo L.H. Bailey en condiciones de estrés salino y déficit de humedad. Asimismo, los efectos se compararon con los de un HMA exótico, con la finalidad de determinar si existen diferencias entre las especies fúngicas inoculadas, ya que la efectividad para incrementar la resistencia al estrés hídrico se ha relacionado con la producción de micelio externo y la frecuencia de colonización de la raíz de cada especie fúngica (Marulanda et al., 2003; Avio et al., 2006).

\section{MATERIALES Y MÉTODOS}

\section{Obtención de inóculos}

Dos inóculos de HMA nativos se obtuvieron a partir de muestras de suelo procedentes de campos salinos abandonados de La Costa de Hermosillo (LCH). El inóculo compuesto por un consorcio de campo (InC) se produjo mediante el cultivo trampa $C$. pepo var. pepo crecido en suelo de $\mathrm{LCH}$ en condiciones de irradiancia controlada y con riego abundante hasta la floración; después de $35 \mathrm{~d}$ de cultivo, el riego se suspendió por $5 \mathrm{~d}$ con la finalidad de aumentar el número de esporas de las morfoespecies asociadas. El inóculo mixto (InA) integrado con dos morfoespecies provenientes de los campos salinos de LCH, se produjo en sorgo (Sorghum bicolor (L.) Moench) como hospedante y crecido con suelo estéril inoculado con ambos tipos de esporas. Estas plantas trampa crecieron a una temperatura y humedad relativa promedio de $25^{\circ} \mathrm{C}$ y $35 \%$, y una densidad de flujo fotónico (DFF) de $800 \mu \mathrm{mol}$ $\mathrm{m}^{-2} \mathrm{~s}^{-1}$.

El InC contenía siete tipos de esporas de HMA: Glomus sp. 1 (103 esporas g $\left.{ }^{-1}\right)$, Glomus claroideum N.C. Schenck \& G.S. Smith (90 esporas $\mathrm{g}^{-1}$ ), Rhizophagus intraradices (N.C. Schenck \& G.S. Smith) C. Walker \& A. Schüßler (34 esporas $\mathrm{g}^{-1}$ ), Pacispora sp. 1 (27 esporas g $\mathrm{g}^{-1}$ ), Glomus sp. 3 (11 esporas $\left.\mathrm{g}^{-1}\right)$, Glomus sp. 4 (10 esporas $\left.\mathrm{g}^{-1}\right)$, Pacispora sp. 2 (7 esporas $\left.\mathrm{g}^{-1}\right)$. El InA contenía dos tipos de esporas: Glomus sp. 1 (42 esporas $\left.\mathrm{g}^{-1}\right)$ y Glomus sp. 4 (24 esporas $\mathrm{g}^{-1}$ ).

\section{Condiciones de cultivo y tratamientos de inoculación}

Se sembraron cinco semillas de C. pepo var. pepo en $5 \mathrm{~L}$ de sustrato estéril (5:2 turba negra:suelo) en abril y septiembre 2009. Las plantas crecieron hasta floración bajo sombrea-

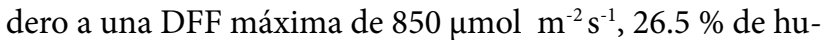
medad relativa y $24{ }^{\circ} \mathrm{C}$ de temperatura ambiental. En cada tratamiento se utilizaron seis repeticiones con tres plantas por maceta.

La inoculación se inició 10 d después de la siembra y cada planta se inoculó con $3 \mathrm{~g}$ de In A, $1.5 \mathrm{~g}$ de InC, 1 $\mathrm{g}$ de $\mathrm{G}$. claroideum procedente de una región templada (Hueyotlipan, Tlaxcala, México) como especie exótica (InG). Se estableció un tratamiento de fertilización química en solución (8 meq $\mathrm{L}^{-1}$ de $\mathrm{N}, 2$ meq $\mathrm{L}^{-1}$ de P y 4 meq $\mathrm{L}^{-1}$ de $\mathrm{K})$ diaria sin HMA (TF), según lo recomendado por SIAP (2009), y un testigo sin fertilización química y sin HMA (T0). En TF y T0 se agregó $1 \mathrm{~g}$ de tierra estéril por planta para igualar el volumen de suelo.

\section{Estrés por déficit de humedad y salinidad}

Se llevaron a cabo tres experimentos independientes, en dos de los cuales se evaluó una condición estresante, salinidad baja o déficit de humedad, una sin estrés, y el otro únicamente con estrés salino alto. En el experimento 1 conducido de abril a junio 2009, el estrés salino bajo se generó con riegos con $\mathrm{NaCl} 40 \mathrm{mM}$, que mantuvo la conductividad eléctrica en $3.8 \pm 1.2 \mathrm{dS} \mathrm{m}^{-1}$. En el segundo experimento efectuado de septiembre a noviembre 2009, las plantas con estrés por déficit de humedad se regaron diariamente con 25 a $50 \mathrm{~mL}$ de agua ( 25 a $40 \%$ de humedad en el suelo). Las plantas sin estrés de los experimentos 1 y 2 se mantuvieron con riego diario de $100 \mathrm{~mL}$ de agua (50 a 60 $\%$ de humedad y $\left.1.4 \pm 0.43 \mathrm{dS} \mathrm{m}^{-1}\right)$. El tercer experimento, conducido simultáneamente con el segundo, se hizo para evaluar únicamente plantas con estrés salino alto, mediante riego con $\mathrm{NaCl} 50 \mathrm{mM}$ que mantuvo la conductividad eléctrica promedio en $4.5 \mathrm{dS} \mathrm{m} \mathrm{m}^{-1}$. El volumen de riego y la concentración de $\mathrm{NaCl}$ para generar la condición de estrés que permitiera crecer las plantas hasta floración se establecieron en experimentos preliminares.

\section{Evaluación de la respuesta}

La biomasa seca total de las plantas por maceta se cuantificó después de deshidratarlas en una estufa (Blue M Stabil-Therm, modelo D-5226-Q, Estados Unidos) a 60 ${ }^{\circ} \mathrm{C}$ por $72 \mathrm{~h}$. La fluorescencia de la clorofila se midió a una temperatura ambiental de 25 a $28{ }^{\circ} \mathrm{C}$, con un fluorímetro Handy PEA (Hansatech Instruments Ltd., Inglaterra), con tres diodos emisores de luz de alta intensidad, longitud de onda de $650 \mathrm{~nm}$ y sobre una superficie foliar de $4 \mathrm{~mm}$ de diámetro. Tres mediciones por tratamiento se hicieron en la misma área foliar de la tercera hoja a partir del meristemo, en intervalos de $0.05,0.1,0.3,2$ y $30 \mathrm{~ms}$, después de mantener la sección de la hoja en oscuridad durante $30 \mathrm{~min}$. 
Las mediciones se hicieron a los 20 y $27 \mathrm{~d}$ después del inicio de la condición estresante. Las variables evaluadas fueron: intensidad de fluorescencia basal $\left(\mathrm{F}_{0}\right)$, máxima intensidad de fluorescencia $\left(\mathrm{F}_{\mathrm{m}}\right)$ y tiempo para alcanzar máxima fluorescencia $\left(\mathrm{T}_{\text {fmax }}\right)$. Con estas variables se calcularon los índices de flujo de fotones absorbidos por unidad de área (ABS/CS), probabilidad de que un fotón absorbido active la cadena de transporte de electrones $\left(\Phi_{\mathrm{OE}}\right)$ y eficiencia con que un electrón es transferido a lo largo de la cadena de transporte de electrones $\left(\psi_{0}\right)$, de acuerdo con Strasser et al. (1995).

La cantidad de glucosa para proveer el C y la energía necesaria para construir el tejido (g glucosa $\mathrm{g}^{-1}$ biomasa seca) se calculó con el calor de combustión $\left(\Delta \mathrm{H}_{\mathrm{C}}\right)$ medido con una bomba calorimétrica Parr (Plain Jakcet, modelo 1341, Estados Unidos), las cenizas (A) en mufla (Thermolyne type modelo 30400, Estados Unidos) y el contenido de nitrógeno (N) con un sistema LECO PF-528 (Estados Unidos). A partir de estas variables se calcularon los costos de construcción (CC) de acuerdo con Carey et al. (1997), con la fórmula $C C=\left[\left(0.06968 \Delta H_{C}-0.065\right)\right.$ $(1-A)+0.5359 \mathrm{kN}] / E_{G}$, donde $k$ es el estado de oxidación de sustrato nitrogenado (de +5 nitratos a +3 amonio) y $E_{G}$ la eficiencia en la conversión (0.89) (Williams et al., 1987).
El número total de esporas (NTE), micelio externo y colonización micorrízica $(\mathrm{CM})$, se evaluaron en todas las macetas con el método de tamizado húmedo, con la metodología descrita por Herrera-Peraza et al. (2004). El NTE se calculó con el método de flotación en sacarosa. La $\mathrm{CM}$ se evaluó con tinción con azul de tripano de 50 a 100 $\mathrm{g}$ raíces secas. Para el número de hifas se colocaron 0.02 a $0.025 \mathrm{~g}$ de tamizado en un portaobjetos con glicerina y se cuantificaron las hifas a lo largo de cuatro líneas de la misma longitud uniformemente distribuidas en el cubreobjetos.

\section{Análisis estadístico}

Se usó un diseño experimental completamente al azar, con arreglo factorial 5 (inóculo) x 2 (con y sin estrés), para las variables biomasa seca total, micelio externo, CM, NTE y CC $(\mathrm{P} \leq 0.05)$, para los ensayos de déficit de humedad y de salinidad baja. Las diferencias significativas por la combinación de factores se compararon con base en los errores estándares. Para el caso del experimento 3 (estrés salino alto), las diferencias estadísticas entre medias se determinaron con la prueba de Duncan a una probabilidad de error de $5 \%$. Se utilizó el paquete estadístico NCSS versión 2006 (Hintze, 2006).

Cuadro 1. Efecto del inóculo (In) en plantas de C. pepo var. pepo crecidas en salinidad baja, respecto a peso seco, micelio externo (ME), colonización micorrízica (CM), número total de esporas (NTE) y costos de construcción (CC).

\begin{tabular}{cccccc}
\hline In & $\begin{array}{c}\text { Peso seco } \\
(\mathrm{g})\end{array}$ & $\begin{array}{c}\text { ME } \\
(\mathrm{mg} / \mathrm{g} \text { de suelo })\end{array}$ & $\begin{array}{c}\mathrm{CM} \\
(\%)\end{array}$ & $\begin{array}{c}\text { NTE } \\
\text { (espora/10 } \\
\text { suelo })\end{array}$ & $\begin{array}{c}\text { CC } \\
\text { (g glucosa/g peso seco) }\end{array}$ \\
\cline { 2 - 6 } InA & $7.86 \pm 1.9$ & $51.64 \pm 4.2$ & $28.28 \pm 20.6$ & $138.60 \pm 55.1$ & $0.606 \pm 0.012$ \\
InC & $7.24 \pm 1.3$ & $51.66 \pm 2.9$ & $35.24 \pm 16.0$ & $91.81 \pm 35.9$ & $0.648 \pm 0.009$ \\
InG & $5.02 \pm 0.8$ & $32.79 \pm 2.9$ & $53.04 \pm 31.7$ & $65.20 \pm 29.9$ & $0.696 \pm 0.026$ \\
TF & $4.23 \pm 0.9$ & 0.00 & 0.00 & 0.00 & $0.635 \pm 0.023$ \\
T0 & $4.72 \pm 1.1$ & 0.00 & 0.00 & $0.606 \pm 0.012$ \\
& & \multicolumn{5}{c}{ Con estrés } \\
InA & $5.94 \pm 0.9$ & $35.53 \pm 3.1$ & $31.66 \pm 23.1$ & $93.57 \pm 28.1$ & $0.613 \pm 0.028$ \\
InC & $6.11 \pm 1.3$ & $39.53 \pm 3.4$ & $41.51 \pm 19.6$ & $152.05 \pm 40.7$ & $0.684 \pm 0.005$ \\
InG & $4.79 \pm 1.0$ & $45.96 \pm 11.2$ & $23.09 \pm 11.4$ & $96.49 \pm 19.6$ & $0.709 \pm 0.017$ \\
TF & $4.72 \pm 0.7$ & 0.00 & 0.00 & 0.00 & $0.689 \pm 0.026$ \\
T0 & $5.15 \pm 0.4$ & 0.00 & 0.00 & 0.00 & $0.688 \pm 0.018$ \\
& & & Significancia $(\mathbf{P}<\mathbf{0 . 0 5})$ & \\
Estrés (E) & 0.103 & $0.018^{*}$ & 0.347 & 0.210 & $0.000^{*}$ \\
Inóculo (I) & $0.000^{*}$ & $0.000^{*}$ & $0.000^{*}$ & $0.000^{*}$ & $0.000^{*}$ \\
Ex I & $0.043^{*}$ & $0.000^{*}$ & 0.067 & $0.000^{*}$ & $0.000^{*}$
\end{tabular}

Los valores son medias de seis repeticiones \pm error estándar. T0 = sin HMA ni fertilización; TF = fertilizado químicamente; InG = inóculo exótico de $G$. claroideum; In A = inóculo mixto con dos especies nativas del desierto Sonorense; InC = consorcio de campo salino del desierto Sonorense. 


\section{RESULTADOS}

La inoculación de HMA nativos aumentó el peso seco total de calabaza crecida con estrés salino bajo, en comparación con los demás tratamientos, y también elevó el micelio externo y el número total de esporas (Cuadro 1). La colonización fue mayor con InG (53\%) vs. InA (28 \%) e InC $(35 \%)$. Los costos de construcción fueron mayores con InG (0.69 $\left.\mathrm{g}_{\text {de glucosa }} \mathrm{g}^{-1} \mathrm{ps}\right)$ y menores con InA (0.6 g de glucosa $\left.\mathrm{g}^{-1} \mathrm{ps}\right)$.

Los inóculos de HMA nativos incrementaron $(\mathrm{P} \leq 0.05)$ la biomasa seca total de las plantas estresadas, a 5.1 y 4.8 $\mathrm{g}$ con InA e InC respectivamente, en comparación con los demás tratamientos $(<4.5 \mathrm{~g})$. El micelio externo producido por InA e InC superó en más de $10 \mathrm{mg} / 10 \mathrm{~g}$ de suelo que el micelio de la especie exótica (InG). Con InA tendió a haber mayor número total de esporas y una colonización superior a $30 \%$, que con InC e InG. Los menores costos de construcción correspondieron a T0 (0.78 g de glucosa $\left.\mathrm{g}^{-1} \mathrm{ps}\right)$, seguido por los tratamientos con HMA nativos o

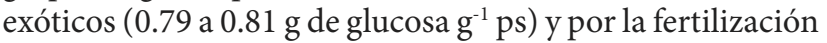
química (0.86 g de glucosa $\mathrm{g}^{-1} \mathrm{ps}$ ), como se muestra en el Cuadro 2.
En plantas con estrés salino alto e inoculadas con los HMA nativos InA e InC (Cuadro 3), el peso seco total fue superior a $6 \mathrm{~g}$, mientras que con los demás tratamientos menor a 5.5 g. En cuanto a las características de micorrización, el micelio externo más abundante se obtuvo con InC $(61 \mathrm{mg} / 10 \mathrm{~g}$ de suelo $v s .42-46 \mathrm{mg} / 10 \mathrm{~g}$ de suelo con InA e InG). La colonización micorrízica fue similar con los tres inóculos de HMA ( $\approx 20 \%)$, pero en el número total de esporas InG, superó en $25 \%(\mathrm{P} \leq 0.05)$ a InA que tuvo la menor cantidad. Los costos de construcción más bajos se registraron con InC e InG $\left(0.72 \mathrm{~g}^{\text {de glucosa }} \mathrm{g}^{-1} \mathrm{ps}\right)$ y los mayores con InA ( $0.78 \mathrm{~g}$ de glucosa $\left.\mathrm{g}^{-1} \mathrm{ps}\right)$, este último similar al T0 sin fertilización y sin inoculación.

En cuanto a la fotoquímica registrada después de 20 d con déficit de humedad, los valores más altos de $\Phi_{O \mathrm{E}} \mathrm{y}$ $\psi_{0}$ se obtuvieron con T0 e InC, y la menor ABS/CS con TF (Figuras 1a y 1b). En el experimento para evaluar la salinidad baja, los tres índices calculados fueron similares entre tratamientos con inoculación (Figuras 1c y 1d). Con salinidad alta, InA y TF presentaron los valores mayores de eficiencia $\left(\psi_{0}\right)$ y de probabilidad para que los fotones y electrones lleguen a la cadena de transporte de electrones $\left(\Phi_{\mathrm{OE}}\right)$ a los $20 \mathrm{~d}$ de iniciado el estrés, mientras estos parámetros fueron bajos con T0 e InG. Asimismo la ABS/

Cuadro 2. Efecto del inóculo (In) en plantas de C. pepo var. pepo sometidas a déficit de humedad, con respecto a peso seco, micelio externo (ME), colonización micorrízica (CM), número total de esporas (NTE) y costos de construcción (CC).

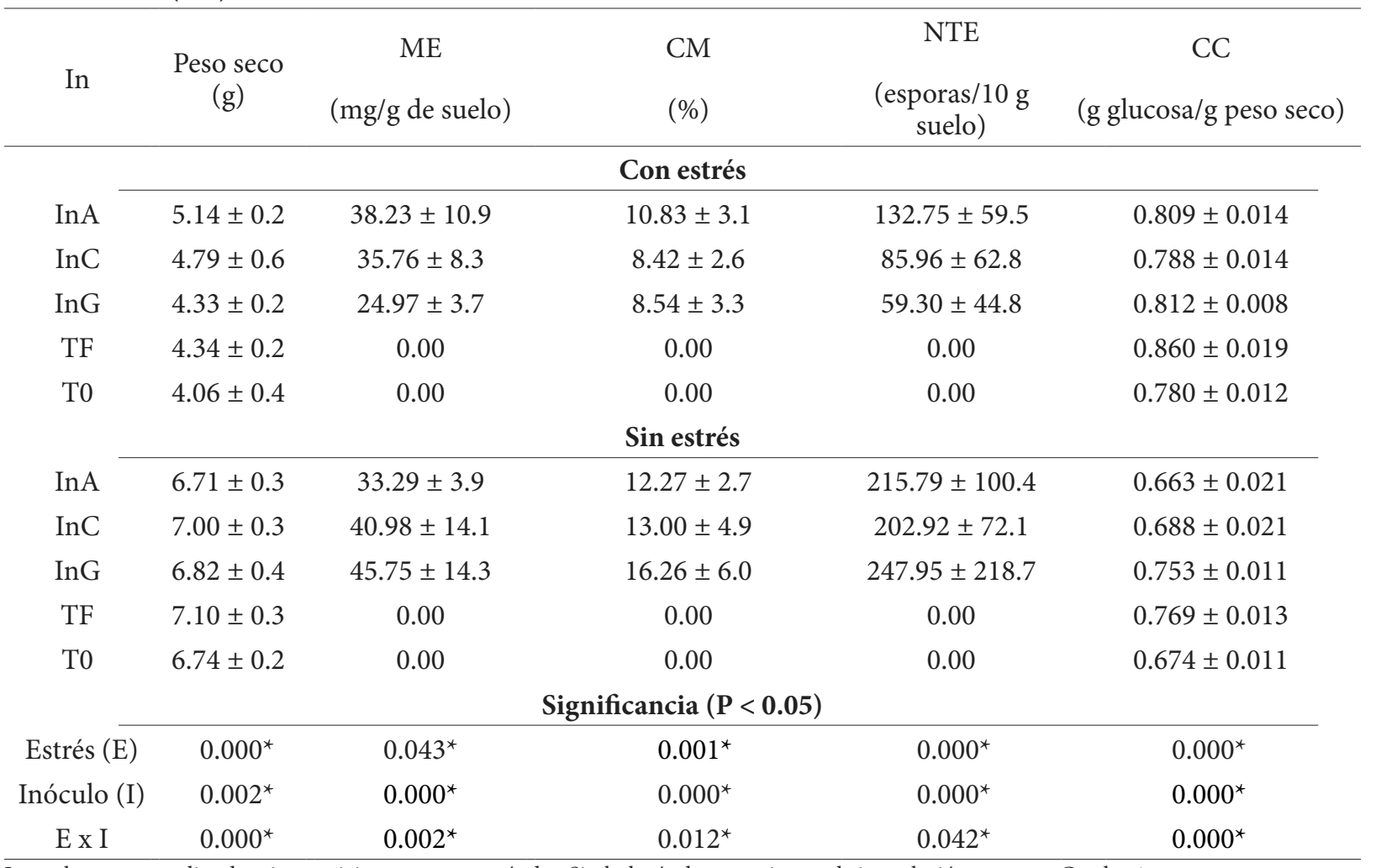

Los valores son medias de seis repeticiones \pm error estándar. Simbología de tratamientos de inoculación como en Cuadro 1. 
Cuadro 3. Efecto del inóculo (In) en C. pepo var. pepo aplicando salinidad alta. Las variables evaluadas fueron: peso seco, micelio externo (ME), colonización micorrízica (CM), número total de esporas (NTE) y costos de construcción (CC).

\begin{tabular}{|c|c|c|c|c|c|}
\hline In & $\begin{array}{l}\text { Peso seco } \\
\text { (g) }\end{array}$ & $\begin{array}{c}\text { ME } \\
(\mathrm{mg} / \mathrm{g} \text { de suelo) }\end{array}$ & $\begin{array}{l}\text { CM } \\
(\%)\end{array}$ & $\begin{array}{c}\text { NTE } \\
\text { (esporas/10 g suelo) }\end{array}$ & $\begin{array}{c}\text { CC } \\
\text { (g glucosa/g peso seco) }\end{array}$ \\
\hline In $\mathrm{A}$ & $6.1 \pm 0.7 \mathrm{ab}$ & $46.2 \pm 21.9 \mathrm{~b}$ & $18.4 \pm 8.0 \mathrm{a}$ & $232 \pm 94 b$ & $0.783 \pm 0.012 \mathrm{a}$ \\
\hline InC & $6.9 \pm 0.5 \mathrm{a}$ & $61.4 \pm 18.7 \mathrm{a}$ & $20.1 \pm 4.3 \mathrm{a}$ & $170 \pm 67 c$ & $0.720 \pm 0.015 c$ \\
\hline In $G$ & $4.8 \pm 0.9 c$ & $42.8 \pm 15.0 \mathrm{~b}$ & $22.3 \pm 6.9 \mathrm{a}$ & $316 \pm 175 \mathrm{a}$ & $0.720 \pm 0.004 c$ \\
\hline $\mathrm{TF}$ & $4.7 \pm 0.6 c$ & $0.00 \mathrm{c}$ & $0.00 \mathrm{~b}$ & $0.00 \mathrm{~d}$ & $0.747 \pm 0.013 b c$ \\
\hline T0 & $5.4 \pm 0.8 \mathrm{bc}$ & $0.00 \mathrm{c}$ & $0.00 \mathrm{~b}$ & $0.00 \mathrm{~d}$ & $0.762 \pm 0.020 \mathrm{ab}$ \\
\hline
\end{tabular}

Diferente literal en una columna indica diferencias significativas (Duncan, 0.05 ). Los valores son medias de seis repeticiones \pm error estándar. Simbología de tratamientos de inoculación como en Cuadro 1.

CS con los inóculos de HMA nativos y T0 fue mayor que con TF, y los valores más bajos fueron con InG (Figura 1e).

A los 27 d de estrés (Figura 2) las plantas T0 con déficit de humedad tuvieron los índices más bajos de $\psi_{0} \mathrm{y} \Phi_{0 \mathrm{E}} \mathrm{y}$ los mayores con InA, mientras que la ABS/CS fue mayor en T0 que en los otros cuatro tratamientos (Figura 2a). Sin déficit de humedad se observó que con InA e InC la probabilidad de que un fotón absorbido se mueva a la cadena de transporte de electrones $\left(\Phi_{\text {OE }}\right)$ y la eficiencia con que un electrón sea transferido a la cadena de transporte de electrones $\left(\psi_{0}\right)$, fueron menores que en InG, TF y T0, pero la cantidad de fotones absorbidos por unidad de área (ABS/ CS) fue mayor o similar (Figura 2b).

Con salinidad baja los valores de $\Phi_{0 \mathrm{E}} \mathrm{y} \psi_{0}$ fueron mayores en las plantas estresadas que en las no estresadas (Figuras 2 c y $2 \mathrm{~d}$ ), pero en las inoculadas con InA la eficiencia con que un electrón sea transferido a la cadena de transporte de electrones fue mayor y la cantidad de fotones absorbidos menor que en los otros cuatro tratamientos (Figura 2d). Con salinidad alta $\Phi_{0 \mathrm{E}} \mathrm{y} \psi_{0}$ fueron similares entre tratamientos de inoculación, excepto con InA en la que ambos parámetros disminuyeron. La ABS/CS fue mayor a 1000 unidades en las plantas inoculadas con HMA y en T0, mientras que con TF se registró el valor menor (Figura 2e). Las diferencias en los índices calculados para evaluar la fotoquímica fueron más pronunciadas con el estrés salino alto que con el déficit de humedad y la salinidad baja (Figura 1e y 2 e).

\section{DISCUSIÓN}

Los HMA nativos del desierto Sonorense produjeron más micelio, lo que se relacionó con mayor incremento del peso seco total en plantas crecidas con salinidad baja y déficit de humedad (Cuadros 1 y 2). El micelio externo producido por los HMA modifica la retención de agua en el suelo y por tanto el estado hídrico y crecimiento de las plantas (Augé et al., 2003; Cho et al., 2005), por lo que en condiciones de estrés hídrico los HMA pueden beneficiar el crecimiento de los tejidos vegetales.
Estudios previos han demostrado que una mayor colonización de las raíces incrementa la demanda energética, por lo que se espera que los costos de construcción (CC) sean mayores en plantas con porcentajes altos de colonización (CM) (Peng et al., 1993). Los resultados de los experimentos con salinidad baja y déficit de humedad concuerdan con el comportamiento descrito anteriormente, pues las plantas con déficit de humedad e inoculadas con HMA tuvieron valores similares de CC y CM; en cambio, con salinidad baja esos valores disminuyeron, y el orden de menor a mayor: InA, InC e InG (Cuadros 1 y 2).

La micorrización puede representar una demanda energética a la planta que no es compensada por los beneficios de la asociación, lo cual puede ocurrir cuando los organismos no están adaptados a las condiciones ambientales (Tinker et al., 1994; Grimoldi et al., 2006). En este estudio, C. pepo var. pepo crecida con o sin estrés (salino y déficit de humedad) e inoculada con HMA nativos, presentó mayor tejido vegetal y menores costos que cuando fue inoculada con la especie exótica.

La baja disponibilidad de agua en el suelo limita la asimilación de $\mathrm{CO}_{2}$ al disminuir la apertura de estomas, lo que ocasiona daño oxidativo y altera las reacciones de los Fotosistemas I y II en los cloroplastos (Tambussi et al., 2002; Flexas et al., 2009). Esto puede ser revertido mediante mecanismos de protección con pigmentos y enzimas antioxidantes que disipan el exceso de energía en el cloroplasto e incrementan la captación de agua por el sistema radical (Terzi et al., 2010; Jaleel et al., 2009). Una forma de evaluar las alteraciones en el aparato fotosintético es la medición de fluorescencia de clorofila (Oukarroum et al., 2009). En nuestro estudio se encontró que los tratamientos de inoculación modificaron diferentemente los índices ABS/CS, $\Phi_{0 \mathrm{E}}$, y $\psi_{0}$ entre las plantas con y sin estrés hídrico (Figuras 1a, 1b, 2a y 2b), ya que la presencia de HMA puede promover los mecanismos antioxidantes o la absorción de agua por el desarrollo del hongo. 

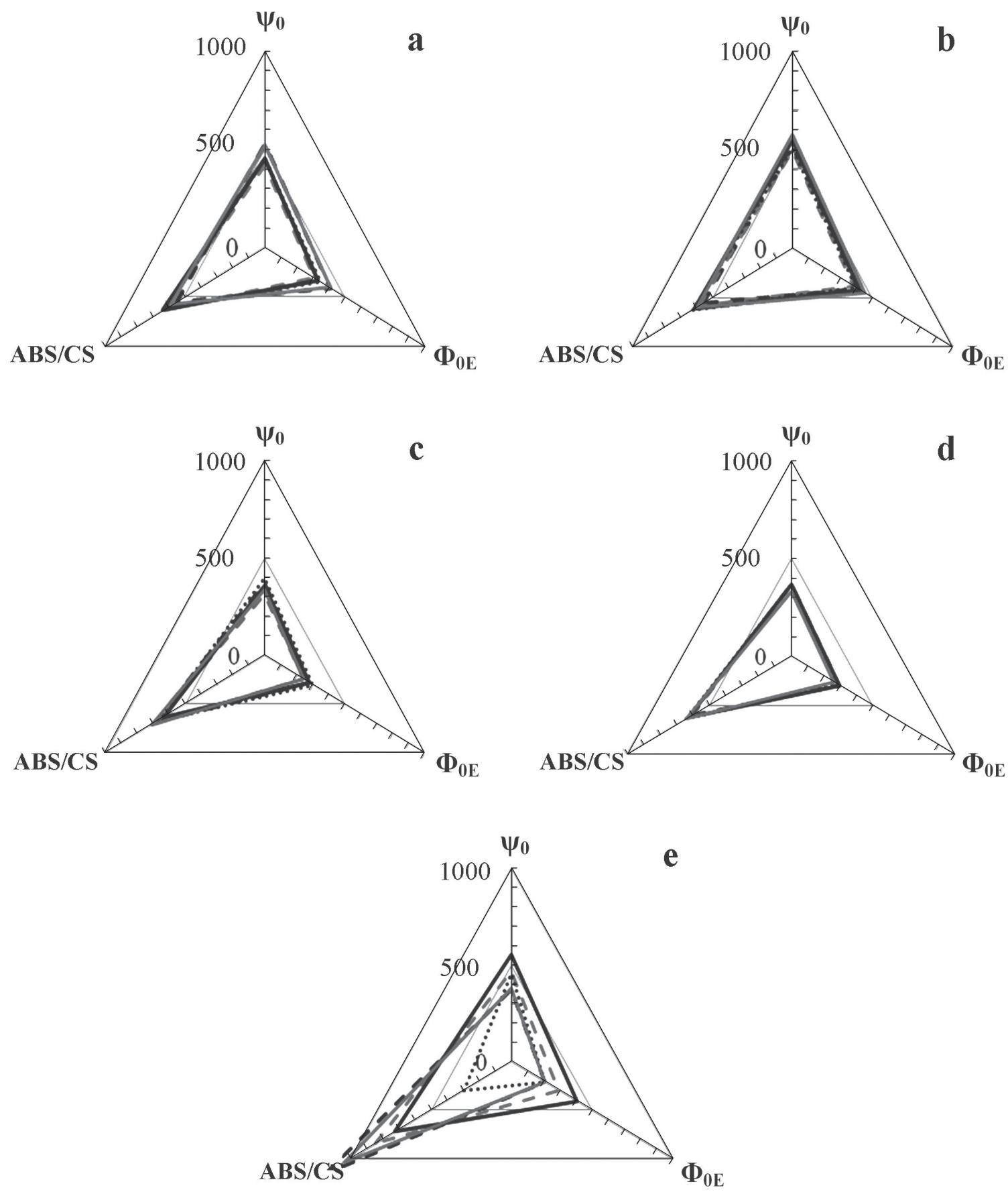

Figura 1. Cambios en la fotoquímica de las plantas después de $20 \mathrm{~d}$ de déficit de humedad (a), sin déficit de humedad (b), salinidad baja (c), salinidad alta (e) y sin salinidad (d). Los ejes indican los fotones absorbidos por unidad de área $(\mathrm{ABS} / \mathrm{CS})$, probabilidad de que un fotón absorbido se mueva a la cadena de transporte de electrones $\left(\Phi_{0 \mathrm{~F}}\right)$ y eficiencia con que un electrón es transferido a la cadena de transporte de electrones $\left(\psi_{0}\right) . \mathrm{T} 0=$ testigo $(-), \mathrm{TF}=$ fertilizado químicamente $(-), \mathrm{InG}=$ inóculo exótico de G. claroideum ( ...... ), InA = inóculo mixto con dos especies nativas del desierto Sonorense ( - - - ), InC = consorcio de campo salino del desierto Sonorense $(-\overline{-}-$ ). 

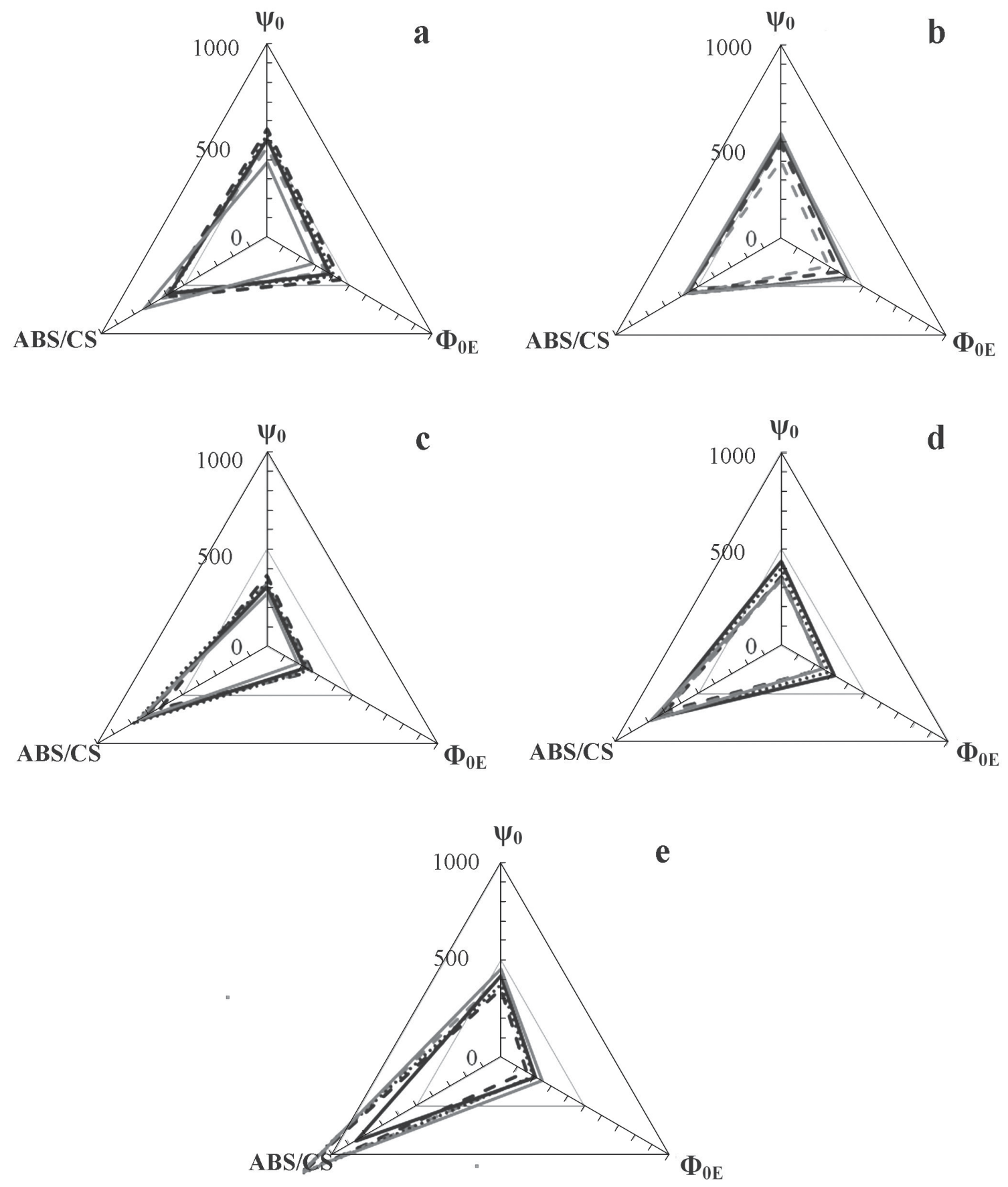

Figura 2. Cambios en la fotoquímica de las plantas después de 27 d de déficit de humedad (a), sin déficit de humedad (b), salinidad baja (c), salinidad alta (e) y sin salinidad (d). Simbología de tratamientos de inoculación y de ejes, como en Figura 1.

Un estudio en alfalfa (Medicago sativa L.) mostró que los HMA benefician a las plantas cuando crecen con limitaciones de agua, porque incrementaron el transporte de electrones, la absorción de energía y la biomasa vegetal (Tsimilli-Michael et al., 2000). En plantas C. pepo con $27 \mathrm{~d}$ de déficit de humedad e inoculadas con InA fue mayor la eficiencia en la transferencia de electrones y la probabilidad de que un fotón absorbido se mueva a la cadena de transporte de electrones, aunque el peso seco disminuyó un poco por efecto del estrés. Además, los costos de construcción fueron menores que InG y TF, lo que podría estar relacionado con incrementos en la captación de energía luminosa y compensación del gasto en la manutención del simbionte. 
Un estudio para evaluar el efecto del $\mathrm{NaCl}$ en los complejos proteicos de los tilacoides mostró que la condición salina afecta la oxidación del agua y los centros de reacción activos y, por tanto, la eficiencia del Fotosistema II (Misra et al., 1999). En C. pepo la salinidad incrementó la absorción de fotones y disminuyó la probabilidad de la incorporación del fotón a la cadena transportadora de electrones, principalmente al final del experimento (Figura 2c). Los cambios fueron mayores con el incremento de la salinidad (Figuras 1e y 2e), similar a lo observado en Vigna radiata (L.) Wilczek y Brassica juncea (L.) Czern. (Misra et al., 2001).

Los cambios en la absorción de fotones, aun la disminución de $\Phi_{0 \mathrm{E}}$ bajo estrés salino (Figuras 1c, 1e, 2c y 2e), pueden estar relacionados con la producción de compuestos reducidos que disminuyen el efecto nocivo de las especies reactivas de oxígeno generadas por hiperosmolaridad, y con la activación por energía luminosa de canales iónicos que regulan el equilibrio osmótico celular (Dajic, 2006).

Los resultados aquí obtenidos en fluorescencia de clorofilas indican que la inoculación de InA incrementó la probabilidad y la eficiencia en el uso de la energía luminosa por las plantas con estrés por déficit de humedad y salino bajo (Figuras 2a y 2c, respectivamente). La inoculación con la mezcla de HMA nativos mejoró el crecimiento de las plantas con costos bajos de construcción, en comparación con InC e InG. Esto sugiere que su inoculación en campo podría disminuir el efecto de la disponibilidad baja de agua y de la salinidad baja en el suelo. Oyetunji et al. (2007) observaron diferencias de la eficiencia fotoquímica y en la tolerancia a la sequía de Manihot esculenta Crantz, en función del HMA asociado, Funneliformis mosseae (T.H. Nicolson \& Gerd.) C. Walker \& A. Schüßler o G. claroideum.

La inoculación con InC en plantas sujetas a estrés salino alto permitió crecimiento mayor, menor costo de construcción y valores de ABS/CS, $\Phi_{0 \mathrm{E}}$ y $\psi_{0}$ mayores o similares a los obtenidos con InA después de 20 y $27 \mathrm{~d}$ de iniciado el estrés (Figuras 1e y 2e). Por ello, el consorcio de campo de los HMA nativos puede ser efectivo para disminuir los efectos nocivos de la salinidad alta, en los cultivos. Los resultados de este estudio confirman que la micorrización modifica la maquinaria fotosintética de las plantas hospederas (Zubek et al., 2009), por lo que sería conveniente un análisis más detallado de los cambios en los mecanismos fotosintéticos debidos a las micorrizas, bajo ciertas condiciones de estrés y en la etapa de producción, particularmente con plantas cultivadas en regiones áridas y semiáridas con salinidad y déficit de agua.

\section{CONCLUSIÓN}

Los HMA nativos del desierto Sonorense promueven el incremento del crecimiento de C. pepo var.pepo sometidas a estrés salino o déficit de humedad, ya que aumentan más la biomasa de las plantas con menores costos de construcción, que con el inóculo exótico de G. claroideum o que con la fertilización química. Tal promoción está relacionada con cambios en la fotoquímica de las plantas que compensan el gasto energético por la transferencia de energía al hongo. Los cambios en los porcentajes de colonización micorrízica están relacionados con el origen de los HMA inoculados y el estrés. El desarrollo mayor de micelio externo del HMA nativo, comparado con el exótico, indica su adaptación a las condiciones de déficit de humedad y salinidad.

\section{AGRADECIMIENTOS}

El apoyo a través del proyecto IAI-CRN II-14, US-NSF (GEO-04523250) para esta investigación sobre HMA. Al M.C. José Llano (UNISON) y M.C. Laura Contreras Angulo (CIAD) por su ayuda en la mediciones. A la M.C. Laura Hernández (Universidad Autónoma de Tlaxcala) por la donación de inóculo de G. claroideum.

\section{BIBLIOGRAFÍA}

Augé R M, J L Moore, K Cho, D M Sylvia, A Al-Agely, A M Saxton (2003) Relating foliar dehydration tolerance of mycorrhizal Phaseolus vulgaris to soil and root colonization by hyphae. J. Plant Physiol. 160:1147-1156.

Avio L, E Pellegrino, E Bonari, M Giovannetti (2006) Functional diversity of arbuscular mycorrhizal fungal isolates in relation to extraradical mycelial networks. New Phytol. 172:347-357.

Black K G, D T Mitchell, B A Osborne (2000) Effect of mycorrhizalenhanced leaf phosphate status on carbon partitioning, translocation and photosynthesis in cucumber. Plant Cell Environ. 23:797-809.

Bhoopander G, K G Mukerji (2004) Mycorrhizal inoculant alleviates salt stress in Sesbania aegyptiaca and Sesbania grandiflora under field conditions. Mycorrhiza 14:307-312.

Carey EV, R M Callaway, E H DeLucia (1997) Stem respiration of ponderosa pines grown in contrasting climates: implications for global climate change. Oecologia 111:19-25.

Cho K, H Toler, J Lee, B Ownley, J C Stutz, J L Moore, R M Augé (2005) Mycorrhizal symbiosis and response of sorghum plants to combined drought and salinity stresses. J. Plant Physiol. 163:517528.

Dajic Z (2006) Salt stress. In: Physiology and Molecular Biology of Stress Tolerance in Plants. K V Madhava Rao, A S Raghavendra, K J Reddy (eds). Springer. Netherlands. pp:41-99.

Flexas J, M Barón, J Bota, J-M Ducruet, A Gallé, J Galmés, M Jiménez, A Pou, M Ribas-Carbó, C Sajnani, M Tomás, H Medrano (2009) Photosynthesis limitations during water stress acclimation and recovery in the drought-adapted Vitis hybrid Richter-110 ( V. berlandieri.V. rupestris). J. Exp. Bot. 60:2361-2377.

García I, R Mendoza, M C Pomar (2008) Deficit and excess of soil water impact on plant growth of Lotus tenuis by affecting nutrient uptake and arbuscular mycorrhizal symbiosis. Plant Soil 304:117-131.

Grimoldi A A, M Kavanová, F A Lattanzi, R Schäufele, H Schnyder (2006) Arbuscular mycorrhizal colonization on carbon economy in perennial ryegrass: quantification by ${ }^{13} \mathrm{CO}_{2} /{ }^{12} \mathrm{CO}_{2}$ steady-state labeling and gas exchange. New Phytol. 172:544-553.

Herrera-Peraza R A, E Furrazola, R Fernandez-Valle, Y Torres (2004) Functional strategies of root hairs and arbscular mycorrhizae in evergreen tropical forest, Sierra del Rosario, Cuba. Rev. CENIC Ciencias Biol. 35:113-123.

Hintze J (2006) NCSS, PASS and GESS. NCSS. Kaysville, USA. (www. NCSS.com).

Jaleel C A, P Manivannan, A Wahid, M Farooq, H J Al-Juburi, R 
Somasundaram, R Panneerselvam (2009) Drought stress in plants: a review on morphological characteristics and pigments composition. Intern. J. Agric. Biol. 11:1560-8530.

Lapointe L, J Molard (1997) Costs and benefits of mycorrhizal infection in a spring ephemeral, Erythronium americanum. New Phytol. 135:491-500.

Lekberg Y, R T Koide (2005) Is plant performance limited by abundance of arbuscular mycorrhizal fungi? A meta-analysis of studies published between 1988 and 2003. New Phytol. 168:189-204.

Marulanda A, R Azcón, J M Ruiz-Lozano (2003) Contribution of six arbuscular mycorrhizal fungal isolates to water uptake by Latuca sativa plants under drought stress. Physiol. Plant. 119:526-53.

Misra A N, S M Sahu, M Misra, N K Ramaswamy, T S Desai (1999) Sodium chloride salt stress-induced changes in thylakoid pigment protein complexes, photosystem II activity and thermoluminescence glow peaks. Z. Naturforsch. 54:640-644.

Misra A N, A Srivastava, R Strasser (2001) Utilization of fast chlorophyll a fluorescence technique in assessing the salt/ion sensitivity of mug bean and Brassica seedlings. J. Plant Physiol. 158:1173-1181.

Oukarroum A, G Schansker, R J Strasser (2009) Drought stress effects on photosystem I content and photosystem II thermotolerance analyzed using $\mathrm{Chl} a$ fluorescence kinetics in barley varieties differing in their drought tolerance. Physiol. Plant. 137:188-199.

Oyetunji O J, I J Ekanayake, O Osonubi (2007) Chlorophyll fluorescence analysis for assessing water deficit and arbuscular mycorrhizal fungi (AMF) inoculation in Cassava (Manihot esculenta Crantz). Adv. Biol. Res. 1:108-117.

Peng S, D M Eissenstat, J H Craham, K Williams, N C Hodge (1993) Growth depression in mycorrhyzal citrus at high-phosphorus supply. Plant Physiol. 101:1063-1071.

Ruiz-Lozano J M (2003) Arbuscular mycorrhizal symbiosis and alleviation of osmotic stress. Mycorrhiza 13:309-317.

SIAP, Servicio de Información Agroalimentaria y Pesquera (2009) Detalle Agrícola. Disponible en: http://www.siap.sagarpa.gob.mx.
(Septiembre 2009).

Smith E, D J Read (2008) Mycorrhizal Symbiosis. Academic Press. London, UK. 815 p.

Strasser R J, A Srivastava, R Govindjee (1995) Polyphasic chlorophyll a fluorescence transient in plants and cyanobacteria. Photochem. Photobiol. 61:32-42.

Tambussi E A, J Casadesus, S Munné-Bosch, J L Araus (2002) Photoprotection in water-stressed plants of durum wheat (Triticum turgidum var. durum): changes in chlorophyll fluorescence, spectral signature and photosynthetic pigments. Funct. Plant Biol. 29:35-44.

Terzi R, A Sağlam, N Kutlu, H Nar, A Kadioğlu (2010) Impact of soil drought stress on photochemical efficiency of photosystem II and antioxidant enzyme activities of Phaseolus vulgaris cultivars. Turkish J. Bot. 34:1-10.

Tinker P B, D M Durall, M D Jones (1994) Carbon use efficiency in mycorrhizas: theory and sample calculation. New Phytol. 128:115 122.

Tsimilli-Michael M, P Eggenberg, B Biro, K Köves-Pechy, I Vörös, R J Strasser (2000) Synergistic and antagonistic effects of arbuscular mycorrhizal fungi and Azospirillum and Rhizobium nitrogenfixers on the photosynthetic activity of alfalfa, probed by the polyphasic chlorophyll $a$ fluorescence transient O-J-I-P. Appl. Soil Ecol. 15:169-182.

Williams K, F Percival, J Merino, H A Mooney (1987) Estimation of tissue construction cost from heat of combustion and organic nitrogen content. Plant Cell Environ. 10:725-734.

Wright D P, D R Read, J D Scholes (1998) Mycorrhizal sink strength influences whole plant carbon balance of Trifolium repens L. Plant Cell Environ. 21:881-891.

Zubek S, K Turnau, M Tsimilli-Michael, R J Strasser (2009) Response of endangered plant species to inoculation with arbuscular mycorrhizal fungi and soil bacteria. Mycorrhiza 19:113-123. 